\title{
Spin decoherence in $n$-type GaAs: the effectiveness of the third-body rejection method for electron-electron scattering
}

\author{
Gionni Marchetti. * Matthew Hodgson ${ }^{\dagger}$ and Irene D’Amico ${ }^{\ddagger}$ \\ Department of Physics, University of York, \\ York, Heslington YO10 5DD, UK
}

(Dated: June 15, 2021)

\begin{abstract}
We study the spin decoherence in n-type bulk GaAs for moderate electronic densities at room temperature using the Ensemble Monte Carlo method. We demonstrate that a technique called "third-body rejection method", devised by Ridley [B. K. Ridley, J. Phys. C: Solid State Phys. 10, 1589, 1977] can be successfully adapted to ensemble Monte Carlo method and used to tackle the problem of the electron-electron contribution to spin decoherence in the parameter region under study, where the electron-electron interaction can be reasonably described by a Yukawa potential. This scattering technique is employed in a doping region where one can expect that multiple collisions may play a role in carrier dynamics. By this technique we are able to calculate spin relaxation times which are in very good agreement with the experimental results found by Oertel et al.[S. Oertel, J. Hübner, M. Oestreich, Appl. Phys. Lett., 93, 13, 2008]. Through this method we show that the electron-electron scattering is overstimated in Born approximation, in agreement with previous results obtained by Kukkonen and Smith [C. A. Kukkonen, H. Smith, Phys. Rev. B, 8, 4601, 1973].
\end{abstract}

\section{INTRODUCTION}

Spintronics is a highly active field that encompasses both fundamental research and practical applications. Its goal is to study and exploit spin-related properties in material, e.g. in metals, semiconductors and semiconductor heterostructures, as well as in more exotic structures such as topological insulators or organic molecules 12 . The coherent transport of spin is a central issue in spintronics. For this reason, the spin transport in $n$-type GaAs with its long electronic spin lifetime ${ }^{3}$, has been recently studied both theoretically ${ }^{4 / 6}$ and experimentally ${ }^{78}$. An important goal is to achieve a clear understanding of spin decoherence phenomena due to the carrier dynamics, and in different doping regimes.

In bulk n-GaAs at high temperatures and for the range of doping densities here considered $\left(n_{\mathrm{e}}\right.$ from $10^{16} \mathrm{~cm}^{-3}$ to $2.5 \cdot 10^{17} \mathrm{~cm}^{-3}$ ), the main source of spin relaxation is the Dyakonov-Perel (DP) mechanism? a type of spin-orbit interaction. This mechanism arises from the bulk inversion asymmetry ${ }^{10}$ giving rise to effective, momentum-dependent magnetic fields. Thus due to collisional events which cause momentum transfer, each electronic spin undergoes a precession around a different direction. Such a kinematics gives rise to spin dephasing.

In previous works ${ }^{11 \mid 12}$ we studied spin transport using the Ensemble Monte Carlo (EMC) method. EMC is a stochastic method devised to solve numerically the Boltzmann equation for charge transport in semiconductors ${ }^{13 / 14}$ which is also suitable for studying spin dynamics $11 / 15$. It is worthwhile to recall here that the EMC method applied to charge transport has provided very accurate estimates of semiconductor material properties, e.g. drift velocities, electron mobilities, etc ${ }^{13}$. However the electron-electron (e-e) interactions do not affect greatly the charge transport calculations, due to the conservation of the total energy and momentum, so they could be generally discarded in the EMC calculations $13 / 16$. This is not the case for spin dephasing where the e-e interactions play an important role as theoretically predicted, e.g. by Refs. 4,6 .

In Ref. 12 we simulated the effect of electron-electron scattering on the spin relaxation time (SRT). We found that the inclusion of the e-e scattering was the key to reproduce the experimental results found by Oertel et al ${ }^{7}$. Our results for $n$-type bulk GaAs at relatively high temperatures ( $280 \mathrm{~K} \leq \mathrm{T} \leq 400 \mathrm{~K})$ and moderate doping concentrations ( $n_{\mathrm{e}}$ from $10^{16}$ to $10^{17} \mathrm{~cm}^{-3}$ ) were in very good agreement with experiments.

However, for electronic densities $n_{\mathrm{e}} \gtrsim 10^{17} \mathrm{~cm}^{-3}$ our calculations overstimated the spin relaxation time. We interpreted this as an effect of the failing of the first order Born approximation (BA) (usually simply referred to as "Born Approximation") for the electron-electron scattering. In fact, the Ensemble Monte Carlo algorithm relies on the Fermi Golden Rule which entails the Born approximation, and the latter is well known to perform less well as an approximation for low energy collisions $17 / 18$. Our analysis of the e-e scattering contribution to the spin relaxation time ${ }^{12}$, indicates that the electron- electron scattering is overestimated. This result depends also on the interelectronic potential adopted in the calculations, modeling the conduction electrons as an electron gas (jellium model). In the parameter region where the random phase approximation (RPA) holds, a Yukawa potential can be used ${ }^{19}$. It is reasonable to assume that for $n_{\mathrm{e}} \gtrsim 10^{17} \mathrm{~cm}^{-3}$ and at temperature $\mathrm{T}=300 \mathrm{~K}$ a Yukawa potential is adequate to picture the screened short-range Coulomb interaction.

In Ref. 12 within this model we observed that the discrepancies between the calculations and the experimental results in Ref. 7 become larger when the electronic den- 
sity increases. In this circumstance the average distance between electrons in the conduction band decreases and thus there are reasons for believing that scattering processes which involve more than two electrons simultaneously may become important. Since the scattering formulae usually used in EMC simulations, see Ref. 13, rely on the assumption of truly two-body processes, in order to improve the agrement with the experimental results it is necessary to include this possible physical effect in the calculations. Here we propose to use a simple method called "third-body rejection" (tbr) introduced by Ridley ${ }^{2021}$. This method takes into account the potential third body in a multiple scattering event by introducing an additional probability factor - the "third-body exclusion factor" - which, by renormalizing the scattering cross-section, assures that the collisions are truly two-body processes.

We find that this method improves our SRT calculations at higher doping densities, bringing very good agreement with the experimental results and therefore significantly improving over Ref. 12 .

\section{ENSEMBLE MONTE CARLO METHOD}

In this section we shall give a brief account of the computational aspects of our study. For more detail we refer to Ref. 12

The Ensemble Monte Carlo method is a semiclassical numerical approach suitable to describe charge and spin transport in semiconductors. It includes a sequence of free flights for each simulated particle whose durations are randomly generated. Each free flight time is terminated by a scattering even 13 . After the collision, the energy and momentum of the particle are updated according to one of the possible scattering mechanisms. The process is repeated until enough data are generated according to the aims of the simulation.

Each free flight time $\tau_{0}$ is given by 13

$$
\tau_{0}=\ln (r) / \Gamma_{\text {tot }}
$$

where $r$ is a random number generated stochastically from a uniform distribution on the interval $(0,1)$ and $\Gamma_{\text {tot }}$ is the total scattering rate which includes all the scattering mechanisms of the system under study. $\Gamma_{\text {tot }}$ is calculated at the beginning of the simulation as a function of the colliding particle energy $y$. In between the scattering events, carriers propagate along a classical trajectory which may be influenced by external forces due to applied electric and/or magnetic fields. In the present work we simulate the dynamics of $N=25,000$ carriers.

EMC allows us to follow the spin dynamics together with the carrier dynamics. During the carriers' free flights the electron spins are considered non-interacting, and each spin undergoes a coherent evolution dictated by the spin-dependent part of the Hamiltonian $\frac{12}{12}$. For the system under consideration, the main source of spin relaxation is spin-orbit interaction due to the bulk inversion asymmetry. This is described by the Dresselhaus Hamiltonian $H_{\mathrm{D}} 10$ 1022

$$
H_{\mathrm{D}}=\hbar \boldsymbol{\Omega}(\mathbf{k}) \cdot \vec{\sigma},
$$

where $\vec{\sigma}=\left(\sigma_{x}, \sigma_{y}, \sigma_{z}\right)$ are the Pauli matrices, and the Larmor precession frequency vector $\boldsymbol{\Omega}(\mathbf{k})$ is

$$
\boldsymbol{\Omega}(\mathbf{k})=\frac{\gamma_{s o}}{\hbar}\left[k_{x}\left(k_{y}^{2}-k_{z}^{2}\right), k_{y}\left(k_{z}^{2}-k_{x}^{2}\right), k_{z}\left(k_{x}^{2}-k_{y}^{2}\right)\right] .
$$

Here $k_{i}$ are the wavevector components along the cubic crystal axes, $i=x, y, z$, and $\gamma_{s o}$ is known as the Dresselhaus coefficient, whose values are determined using different methods. In GaAs, $\gamma_{s o}$ values have been suggested which range from 8.5 to $34.5 \mathrm{eV} \AA^{3} 23$.

We are interested in studying the system at equilibrium, therefore we let the system thermalise for a suitable time ${ }^{12}$, after which we realign the electron spins along the $z$-axis. Afterwards the spins will dephase via the Dyakonov-Perel mechanism whereby each spinor wavefunction is acted upon by the time evolution operator generated by the Hamiltonian $H_{\mathrm{D}}$.

Using EMC we can study the spin dynamics of each carrier from its spinor wavefunction. At any given time we can extract the expectation values of the $S_{x}, S_{y}$ and $S_{z}$ components of the individual electron spin operator $S$ to get the probability for the spin to be aligned along each direction. Because we start from an electronic ensemble fully polarized along the $z$-axis, we focus on the time evolution of the expectation value of the total $z$ component spin operator $S_{z \text {,tot }}$. For each simulation, by plotting $S_{z \text {,tot }}$ against time, and assuming an exponential behaviour, we fit the data from the simulation and extract the corresponded spin relaxation time $\tau_{\mathrm{s}}^{[12}$.

\section{A. Scattering Types And Related Approximations}

We consider spin transport in bulk GaAs at $\mathrm{T}=300$ $K$. The carriers' dynamics are described in the framework of a single parabolic energy band (the central $\Gamma$ valley) which gives rise to an effective isotropic electron mass $m^{*}=0.067 m_{\mathrm{e}}$ where $m_{\mathrm{e}}$ is the bare electron mass. We do not include the valleys $\mathrm{X}$ and $\mathrm{L}$ because the electrons we simulate have energies which give a negligible probability to scatter into these valleys 24 .

The carriers (electrons) in our simulations undergo scattering with longitudinal acoustic (LA) phonons, polar longitudinal optical (LO) phonons, singly-ionized impurities in Brooks-Herring approach 13 and finally with other electrons. Electron-piezoacoustical interactions are not included because they become relevant for GaAs samples only at low temperatures ${ }^{21}$. The scattering rate for the electron-LA phonon collisions is determined by 
the acoustic deformation potential in elastic approximation, as inelastic absorption/emission processes are important only at low temperatures ${ }^{13}$. Due to the space group selection rules, for the electrons in the $\Gamma$ valley there is no deformation potential interaction with the optical phonons ${ }^{24}$. The electron-LO scattering rate (Fröhlich interaction ${ }^{25}$ ) includes absorption and emission processes with a threshold energy of $35 \mathrm{meV}$. Phonons are considered at equilibrium at the lattice temperature T. We use the Fermi Golden rule to calculate the scattering rates at first order for each type of scattering mechanism.

We work within the random phase approximation 19 , which neglects the exchange and correlation effects: we model the screened Coulomb interaction between two charges as in a homogeneous electron gas using the (Yukawa-type) Coulomb potential

$$
V(r)=\frac{\mathrm{e}^{2}}{4 \pi \varepsilon r} e^{-\beta_{\mathrm{TF}} r} .
$$

Here $r=\left|\mathbf{r}_{A}-\mathbf{r}_{B}\right|$ is the distance between the interacting electrons denoted by $A$ and $B$, and $\varepsilon$ is the material dielectric constant, $\varepsilon=12.9 \varepsilon_{0}$ in the case of GaAs. The quantity $\beta_{\mathrm{TF}}$ is called Thomas-Fermi wavevector or "inverse screening length" and is derived in the framework of a finite temperature linearized Thomas-Fermi approximation (LTFA) ${ }^{1226}$. For $n$-type semiconductors with a parabolic band, it is given by the following expression 2728

$$
\beta_{\mathrm{TF}}^{2}=\frac{n_{\mathrm{e}} \mathrm{e}^{2}}{\varepsilon k_{\mathrm{B}} \mathrm{T}} \frac{\mathscr{F}_{-1 / 2}(\eta)}{\mathscr{F}_{1 / 2}(\eta)} .
$$

Here e and $k_{\mathrm{B}}$ are the electron charge and the Boltzmann constant respectively, $\mathscr{F}_{j}$ denotes the Fermi-Dirac integral of order $j, \eta=\mu /\left(k_{\mathrm{B}} \mathrm{T}\right)$ and $\mu$ is the electronic chemical potential. If the RPA holds, then the LTFA is a fair approximation only insofar as we have small disturbances in the electron gas, or equivalently small momentum transfer in Coulomb scattering processes, see Refs. 12 and 29 .

For completeness we should also mention that in the (homogeneous) electron gas model proposed by Pines and Bohm's theory $y^{30}-32$ the Coulomb interaction is split in two contributions: a Yukawa Coulomb scattering between individual electrons and an electron-plasmon interaction. The latter is not included in our calculations because the electron-plasmon scattering becomes important in GaAs for higher electronic concentrations than considered in the present work ${ }^{[13}$.

In order to make the e-e scattering consistent with the Pines and Bohm's model described above, when a carrier undergoes an e-e collision in our EMC simulations, its electron partner is chosen arbitrarly but within a distance of one screening length. This is an improvement

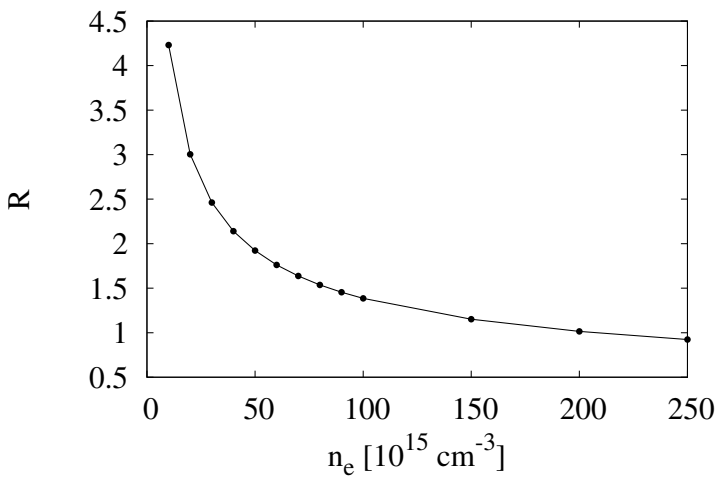

FIG. 1. The inequality given by Eq. (6) against the electron density at $\mathrm{T}=300 \mathrm{~K}$.

over commonly used algorithms which select the second electron from the whole ensemble and with a uniform distribution. Our choice describes much better the locality of a screened electron-electron interaction. Moreover this is consistent with the e-e scattering rate we employ as it follows by assuming a local field theory 33 .

Finally we shall say a few words about the e-e scattering for low energy collisions. In the low energy limit the first order Born approximation for a potential given by Eq. (4) is valid when ${ }^{34}$

$$
\mathrm{R}=\frac{m^{*} \mathrm{e}^{2} \lambda_{\mathrm{TF}}}{4 \pi \varepsilon \hbar^{2}}=\frac{\lambda_{\mathrm{TF}}}{a_{\mathrm{B}}^{*}} \ll 1,
$$

with $a_{\mathrm{B}}^{*}=\left(4 \pi \hbar^{2} \varepsilon\right) /\left(\mathrm{e}^{2} m^{*}\right)$, the effective Bohr radius and $\lambda_{\mathrm{TF}}=1 / \beta_{\mathrm{TF}}$. The inequality (6) is not satisfied for the range of densities considered here, as $\mathrm{R} \lesssim 1$ (see Fig. 11. This confirms the fact that in general the BA is not adequate for low energy processes $\$ 1835$.

By using the phase-shift calculation of electronelectron scattering $\mathrm{g}^{36}$, Kukkonen and Smith ${ }^{17}$ have found that the electron-electron total cross-section in BA for a metal like $\mathrm{Na}$ is overstimated by a factor two. Kukkonen and Smith assumed a scattering potential as in Eq. (4) and included also the antisymmetry of the wavefunction of the colliding carriers. In the case of an electronic gas in a solid, the average interelectronic distance $r_{\mathrm{s}}$ is defined by the relatior ${ }^{29} n_{\mathrm{e}}^{-1}=(4 \pi / 3)\left(r_{s} a_{\mathrm{B}}^{*}\right)^{3}$; for Na its value is 3.96. Therefore we might expect a similar trend in semiconductors with a comparable $r_{\mathrm{s}}$, as in the case here considered.

\section{IMPROVING OVER THE BORN APPROXIMATION: THIRD-BODY REJECTION}

In the following we wish to focus on the density range $1.5 \times 10^{17} \mathrm{~cm}^{-3} \lesssim n_{\mathrm{e}} \leq 2.5 \times 10^{17} \mathrm{~cm}^{-3}$, where the 
RPA is appropriate as $r_{s} \lesssim 1$, see Ref. 12. In this intermediate density range, according to the discussion at the end of the previous section, and to our previous statistical analysis of e-e scattering 12 , we expect the Born approximation to overestimate the e-e scattering cross-section.

The key issue is that the standard theory of scattering assumes that each collision involves only two bodies, while an overestimate of the cross-section, e.g., due to the BA, as in this case, increases the probability of having a third electron within the scattering cross-section. This increased probability of a three-body scattering event can be understood given the usual geometrical interpretation of the cross-section 37 . Furthermore the problem is getting worse when the electronic density $n_{\mathrm{e}}$ increases, as consequently the number of scattering centres increases as well.

The presence of this 'third body' is then not accounted for within the present formalism or, equivalently, the overestimation of the cross-section due to BA invalidates the use of the standard theory of scattering. We wish then to find a method to improve over BA but that is also easily implementable within EMC simulation techniques.

In order to retain the physical picture of a two-body collision for the scattering of an electron with an impurity, and even in the presence of a third carrier, Ridley devised a method called 'statistical screening' or 'thirdbody rejection, 21 . Let us use b to denote the impact parameter (see Fig. 2); then the probability that there is no scattering centre with impact parameter smaller than $b$ is defined by 21

$$
P(\mathrm{~b})=e^{-\pi n_{\mathrm{e}} \mathrm{ab}^{2}}
$$

where $\mathrm{a}=\left(4 r_{s} \mathrm{a}_{\mathrm{B}}^{*} / \pi\right) \sqrt[3]{4 \pi / 3}$ is approximately the average distance between the scattering centres. Using (7), Ridley defines the 'third-body' differential cross-section $\sigma_{\mathrm{AB}}^{(\mathrm{tbr})}(\theta)(\theta$ is the scattering angle $)$, as 21

$$
\sigma_{\mathrm{AB}}^{(\mathrm{tbr})}(\theta)=\sigma_{\mathrm{AB}}^{Y}(\theta) \exp \left(-\pi n_{\mathrm{e}} \mathrm{ab}^{2}\right),
$$

where $\sigma_{\mathrm{AB}}^{Y}(\theta)$ is the Yukawa differential cross-section.

We can give a physical interpretation of $\sigma_{\mathrm{AB}}^{(t b r)}$. In an ideal collision only two particles $A$ and $B$ are involved. Then the total cross-section $\sigma_{\mathrm{AB}}$ is related to the probability that this collision happens. For a given energy of the colliding particle $A$, the effective area determined by $\sigma_{\mathrm{AB}}$ can be roughly thought to be a measure of the tendency of $A$ and $B$ to interact ${ }^{18}$, see top of Fig. 2 .

Let us now add a third-body $C$ to the system, see bottom figure in Fig. 2. Clearly the presence of a new potential scattering centre $C$ for the colliding particle $A$ affects its collision with $B$ and hence reduces the subsystem $A+B$ scattering probability. According to Ridley's formula, Eq. (8), this effect is taken into account by a
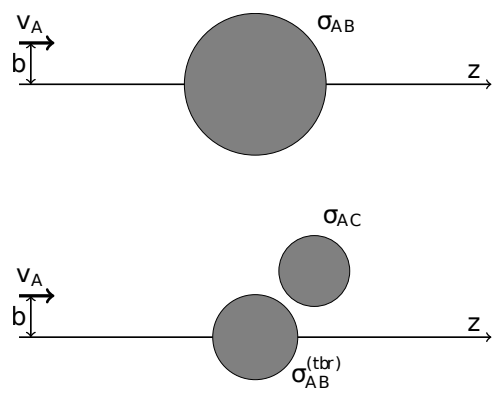

FIG. 2. (Top) Sketch of the cross-section for a collisional event between two particles $A$ and $B$. (Bottom) As above but in the presence of a third body $C$.

reduction in the scattering probability for the the subsystem $A+B$. Thus the total cross-section $\sigma_{A B}$ is now given by $\sigma_{A B}^{(t b r)}$ through the Eq. 8 integrated over the scattering angle interval $[0, \pi]$, as colliding particles are considered distinguishable. We note that in the EMC method the carriers are treated as semiclassical, distinguishable particles. Therefore we shall employ Ridley's method for correcting the scattering in the electron-electron collisions.

\section{A. Embedding the Third-Body Rejection Method in EMC Simulations}

For the third-body rejection method, the scattering rate $\widetilde{w}_{\text {ee }}^{\text {(tbr) }}$ was derived by Van de Roer and Widdershoven ${ }^{38}$ as

$$
\widetilde{w}_{\mathrm{ee}}^{(\mathrm{tbr})}(v)=\frac{v}{\mathrm{a}}\left[1-\exp \left(-\frac{\mathrm{a} w_{\mathrm{ee}}(v)}{v}\right)\right],
$$

where $v$ (group velocity) is the speed associated to the relative motion of the colliding carriers. We shall use Eq. (9) for the e-e scattering rate in order to take into account the presence of a third carrier ${ }^{39}$. Notice also that Eq. 90 implies that $\widetilde{w}_{\mathrm{ee}}^{(\mathrm{tbr})}$ is always smaller than $w_{\mathrm{ee}}$.

In the following we are guided by analogy and computational simplicity; a direct implementation of Eq. (9. in EMC would be very computationally expensive 40 . Firstly as typical in the EMC method, we consider the e-e collisions as independent from the other scattering mechanisms. Because we study the electron system at equilibrium, we can consider the average properties of a typical carrier. Then we can interpret $\mathrm{a} / v$ as a typical time of free flight, which in EMC is given by the inverse of the (average) scattering rate $w_{\text {ee }}(v)$. Then in analogy to Eq. (8), we propose to substitute the probability $w_{e e}(v)$ for $\mathrm{a} / v$, obtaining the following relation in the 


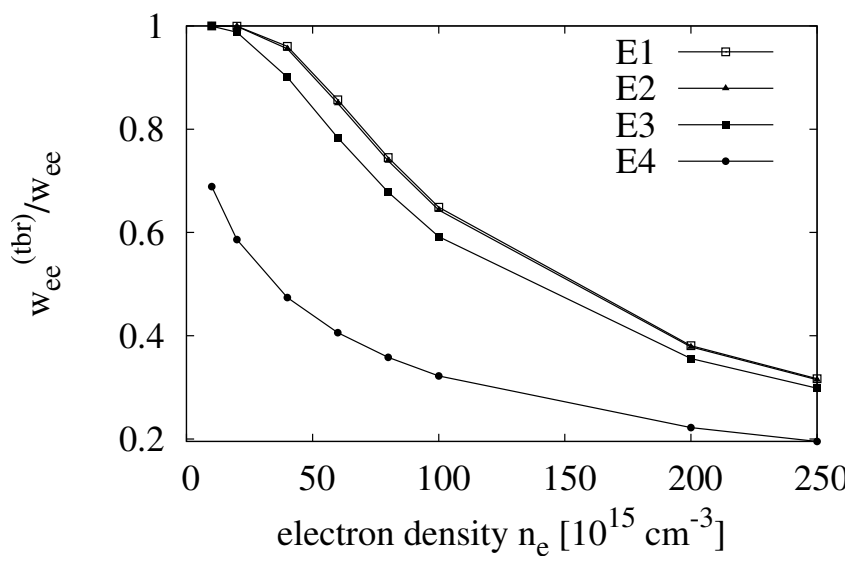

FIG. 3. The ratio $w_{\text {ee }}^{(t b r)} / w_{\text {ee }}$ calculated from Eq. 10 against the electron density at room temperature for four values of the energy: $E 1=10^{-3} \times E_{\mathrm{th}}, E 2=10^{-2} \times E_{\mathrm{th}}, E 3=10^{-1} \times$ $E_{\mathrm{th}}$ and $E 4=E_{\mathrm{th}} . E_{\mathrm{th}}$ is the thermal energy associated to the relative motion.

carrier's relative energy $E_{\text {rel }}$

$$
w_{\mathrm{ee}}^{(\mathrm{tbr})}\left(E_{\mathrm{rel}}\right)=w_{\mathrm{ee}}\left(E_{\mathrm{rel}}\right)\left[1-e^{\left(-\frac{\mathrm{a} m^{*} w_{\mathrm{ee}}\left(E_{\mathrm{rel}}\right)}{\sqrt{2 E_{\mathrm{rel}}}}\right)}\right] .
$$

Consistent with Eq. (8), the tbr scattering probability includes now a weighting term (the term in brackets). Moreover this algorithm is easily implemented in our code because it introduces a mere flag in our EMC electron-electron algorithm.

From Eq. 10 we expect that $w_{\mathrm{ee}}^{(\mathrm{tbr})}$ diminishes the e-e scattering rate with respect $w_{\text {ee }}$. In order to ascertain how much this reduction is, in Fig. 3 we plot the ratio $w_{\mathrm{ee}}^{(t b r)} / w_{\mathrm{ee}}$ against the electron density $n_{\mathrm{e}}$ at temperature $\mathrm{T}=300 \mathrm{~K}$ for different energies associated to the relative motion of the colliding electrons. Here $E_{\text {th }}=(3 / 4) k_{\mathrm{B}} \mathrm{T} \sim 18.75 \mathrm{meV}$ is the electron thermal energy associated to the relative motion.

In Fig. 3 it is evident that the tbr method reduces the e-e scattering rate strongly at higher electronic concentrations. For the thermal carriers in the density range $n_{e}=1.5 \cdot 10^{17}$ to $2.5 \cdot 10^{17} \mathrm{~cm}^{-3}$ the reduction is about $70 \%$ to $80 \%$ while it is $50 \%$ to $70 \%$ for energies smaller than $0.1 E_{\mathrm{th}}$.

Notice that, for the electron densities $n_{e} \lesssim 1.5 \times$ $10^{17} \mathrm{~cm}^{-3}$, the RPA starts to break dowr 12 . This implies that the electron-electron interaction may no longer be considered as a Yukawa-type potential and therefore may fail to model the actual interelectronic potential in that doping region. In this regime the use of the tbr method would not be justified.

\section{COMPARISON WITH EXPERIMENTS}

In this section we present our numerical results for the spin relaxation time $\tau_{\mathrm{s}}$, and compare them to the available experimental data.

Apart from assuming an exponential decay of the total spin polarization in the $z$-direction, we note that our simulations have no fitting parameters. In particular the spin orbit coupling value used is not fitted, but we use the value suggested by Oertel et al ${ }^{7}$ for their experimental data: $\gamma_{\mathrm{so}}=21.9 \mathrm{eV} \AA^{3}$.

In Ref. 12 we observed that, when we include e-e interaction as described in Sec. II A our results for densities $10^{16} \mathrm{~cm}^{-3} \lesssim n_{\mathrm{e}} \lesssim 10^{17} \mathrm{~cm}^{-3}$ are in very good agreement with the experimental data (empty square symbols) reproduced in Fig. 4. In Ref. 12 we interpreted these results as an accidental cancellation of the effects of the Born approximation, see Sec. IIIA, and the use of the Yukawa potential, i.e. overestimation and underestimation of the e-e scattering contribution to the motional narrowing effect ${ }^{22}$ respectively.

However, at higher densities, our results from Ref. 12 (diamonds, Fig. 4) start to overestimate the experimental data for $\tau_{\mathrm{s}}$, reaching $\sim 20 \%$ overestimate when $n_{\mathrm{e}}=$ $2.5 \times 10^{17} \mathrm{~cm}^{-3}$.

Let us assume for a moment that all the other scattering mechanisms give a reasonable contribution to the motional narrowing (we shall return to this issue in Sec Vp. We speculate that the overestimate of $\tau_{\mathrm{s}}$ for $n_{\mathrm{e}} \gtrsim 10^{17} \mathrm{~cm}^{-3}$ is again an effect of the BA we employ for the e-e scattering, but now the validity of RPA $\left(r_{\mathrm{s}} \lesssim 1\right)^{19}$ in this electron density region says that in general the Yukawa potential is more suitable for modeling the e-e interaction, so no accidental cancellation is possible. In the following we shall then check whether including corrections due to third-body-rejection to the e-e interaction improves our results.

In Fig. 4 we compare the experimental results from Ref. 7 with our calculations of the spin relaxation times including e-e interactions with $\left(\tau_{\mathrm{s}}^{\mathrm{ee}, \mathrm{tbr}}\right)$ and without $\left(\tau_{\mathrm{s}}^{\mathrm{ee}}\right)$ corrections due to third-body rejection. Following the trend in Fig. 3, the reduction of the e-e scattering rate due to the inclusion of third-body rejection causes a reduction of the spin relaxation times at all densities, which, for the range of densities studied, becomes more significant with increasing density.

Looking at Fig. 4 we observe that, at relatively high densities, when the use of the tbr technique is justified, $\tau_{\mathrm{s}}^{e e, t b r}$ significantly improves over $\tau_{\mathrm{s}}^{\mathrm{ee}}$, giving results within the experimental error bars for $1.5 \times 10^{17} \lesssim n_{\mathrm{e}} \lesssim$ $2.5 \times 10^{17}$.

However for decreasing densities we see that $\tau_{\mathrm{s}}^{\text {ee,tbr }}$ departs from the experimental results, underestimating $\tau_{\mathrm{s}}$, and significantly so for the density range where $\tau_{\mathrm{s}}^{\text {ee }}$ has a good agreement with the experimental data and the 


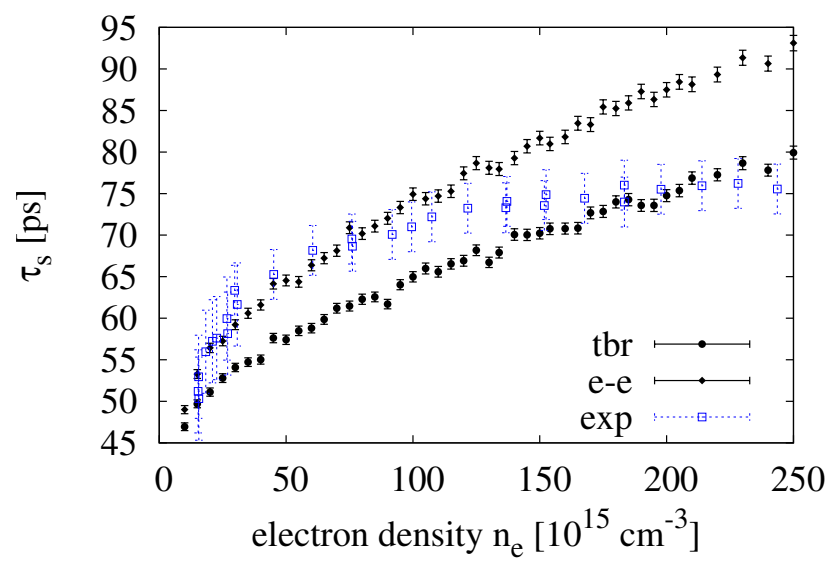

FIG. 4. Spin relaxation time versus electron density. All data include e-e scattering, with (solid circles), and without (solid diamonds) third-body rejection corrections. Parameters: $N=25,000, T=300 \mathrm{~K}$, and $\gamma_{\text {so }}=21.9 \mathrm{eV} \AA^{3}$. The experimental data from Ref. 7 are plotted as well (empty square symbols).

use of tbr is not justified.

\section{DISCUSSION}

In this section we discuss our numerical results in the region $n_{\mathrm{e}} \gtrsim 1.5 \times 10^{17} \mathrm{~cm}^{-3}$ where we expect that our model is reasonably closer to the real physical system.

All the scattering mechanisms we included in our calculations, contribute to the motional narrowing effect, and in turn to the spin relaxation times $\tau_{\mathrm{s}}$. The electronphonon interactions which we consider unscreened ${ }^{41}$, are given by the electron-LO scattering and the electron-LA scattering. Both are correctly estimated; in fact the scattering rates we use are well established in the literature and have given quantitatively accurate results in studies of charge transport ${ }^{13 / 21}$. Furthermore the electron-LA scattering is negligible at room temperature.

The e-i scattering, similarly to the e-e scattering, could be expected to be, due to the low energies involved, also beset by the Born approximation. Moreover given that $n_{\mathrm{i}}=n_{\mathrm{e}}, n_{\mathrm{i}}$ being the doping concentration, one might expect that there is also the need for the tbr correction of the e-i scattering; indeed this method was firstly applied to the electron-impurity collisions 21 .

In the following we shall explain why this is not the case. Discarding all the other scattering mechanisms Meyer and Bartoli performed the phase-shift calculations 42 of the $\mathrm{e}-\mathrm{i}$ scattering contribution to the electron mobility for $n$-type GaAs and then compared it to the mobility obtained using the e-i scattering in BA (Brooks-Herring approach) 43 . At room temperature in the doped region of interested, they found a very good

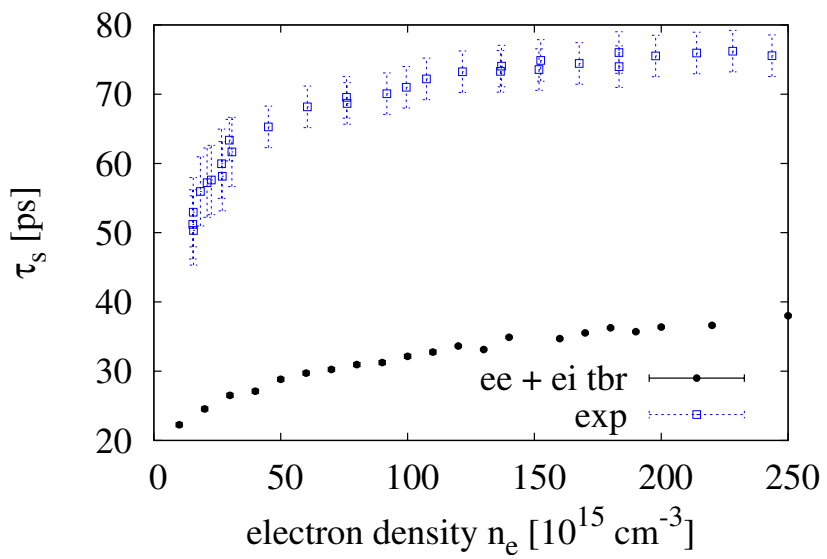

FIG. 5. Spin relaxation time versus electron density. The curve with solid circles represent the data from simulations when the tbr method is applied to both electron-electron and electronimpurity scattering. Parameters: $N=25,000, T=300 \mathrm{~K}$, and $\gamma_{s o}=21.9 \mathrm{eV} \AA^{3}$. The experimental data from Ref. 7 are plotted as well (empty square symbols).

agreement between the two methods. This implies that we can safely assume that the e-i scattering is quite accurate when estimated in BA approximation and for the densities of interest. From this result, recalling that the tbr method would diminish the scattering probability (see Eq. (8) or equivalently Eq. (9)), it is evident that the tbr correction is unnecessary for e-i scattering. This is confirmed a posteriori by the good agreement of our calculations with the experimental data, as shown in Fig. 4.

We note that if we apply the tbr method to both e-e and e-i collisions, we are no longer able to reproduce the experimental data from our simulations, see Fig. 5 Now the spin relaxation times (solid circles) are understimated to a great degree (more than $50 \%$ in some cases). These data show that the overall amount of the Coulomb scattering rate in our numerical simulations, is also decreased as we recover results similar to the case in which e-e interactions are completely neglected, see Ref. 12. Notice that the collisional energy involved in e-e collisions is smaller than the one available in the electron-impurity scattering processes because part of the energy is absorbed by the center of mass's motion ${ }^{44}$. This small collisional energy difference between the Coulomb scattering processes may be then the main cause of the overstimate for the spin relaxation times obtained from our calculations due to the electron-electron scattering in Born approximation.

The close relation between the motional narrowing phenomenon and the collisional regime, in addition with the good agreement of our numerical results via the thirdbody rejection method applied to the electron-electron scattering only, contributes to confirm that the e-e collision rate is overstimated in BA. A quantitative analysis 
of the overestimate can be achieved only evaluating the phase-shifts of the electron-electron scattering for the parameter set of interest, i.e., $n_{\mathrm{e}}, \mathrm{T}, \beta_{\mathrm{TF}}$ and the collisional energy range of the carriers involved in the simulations.

We wish to make some additional remarks on the limits of the physical model we adopt in the present work. First of all, the finite temperature LTFA we use to estimate the screening effects is consistent with a static interaction. This is the case for the electron-impurity interaction where the center of mass of the two-body system can be considered at rest. In an electron-electron interaction because the center of mass moves with speed $v_{c . m}$ in the dielectric medium, the screening has also a frequency dependence $\omega=\mathbf{q} \cdot \mathbf{v}_{\mathbf{c} . \mathbf{m}}$ where $\mathbf{q}$ is the momentum transfe ${ }^{45}$. Then the current results could be improved by including the frequency dependent dielectric function $\epsilon\left(q, \omega=\mathbf{q} \cdot \mathbf{v}_{\mathbf{c} . \mathbf{m}}, \mathrm{T}\right) 19$.

Also we conjecture that quantum intereference in the electron-electron collisions starts to play a role in this regime. Indeed two colliding electrons are in principle indistinguisable fermions. The signature of these effects can be inferred from the Fermi temperature $T_{F}$ which for the region of interest roughly varies from $178 \mathrm{~K}$ to 250 $\mathrm{K}$; a comparison of these values with $\mathrm{T}=300 \mathrm{~K}$ shows that our system is in an intermediate regime, $\mathrm{T} \gtrsim \mathrm{T}_{\mathrm{F}}$, and therefore neglecting the antisymmetry of the colliding electrons's wavefunction, which must include triplet and singlet spin states, might affects our numerical results.

This fact might then explain the flattening of the experimental data in Fig. 4, while the monotonically increasing behaviour of the curves $\tau_{\mathrm{s}}^{\mathrm{ee}}$ and $\tau_{\mathrm{s}}^{\mathrm{ee}, \text { tbr }}$ obtained from our calculations is instead consistent with a nondegenerate regime $\left(\mathrm{T} \gg \mathrm{T}_{\mathrm{F}}\right)$ where the electron-electron scattering rate $w_{\mathrm{ee}} \propto n_{\mathrm{e}}^{\sqrt[6]{46}}$. Therefore we should expect that the quantum mechanical interference due to the fermionic nature of the carriers, diminishes the e-e scattering contribution to the spin relaxation times $\tau_{\mathrm{s}}$ when $n_{\mathrm{e}}$ is relatively high. Anyway the system is far from the degenerate regime in which Pauli principle dominates making the electron-electron collision negligible, as it is typical in the metals. Instead what we guess is happening in the physical system, is analogue to what one usually observes, for instance, in the angular distribution of $\alpha-\alpha$ scattering at relatively low energy $\sim 150 \mathrm{KeV}$, where the quantum interference diminishes the Coulomb scattering for certain values of the scattering angles 47 .

\section{CONCLUSIONS}

We have shown that the third-body rejection method can be successfully employed for studying spin decoherence in semiconductors. With this tool we have been able to obtain a good agreement of our calculations with the experimental data in the doping region where its use can be justified on a physical basis. The third-body method handled quite well the intertwined effects of the Born approximation and a multiple scattering regime.

This little known technique was already successfully employed in the calculations of the mobility in the case of the electron-impurity scattering. Thus we think that this method, based upon a simple physical insight, deserves more attention, and in particular a better understanding of its relation with the Born approximation.

Further work could include the study of quantum intereference due to the direct and exchange transitions $s^{48}$ of the colliding electrons. This could be done, for instance, using the non-relativistic Mott scattering formula 49 .

Additionally the dynamical screening $4 \sqrt{45}$ and nonparabolicity of the conduction band ${ }^{13}$ could be included in the model; however we suspect that the relative improvements might be largely shadowed by the failing of the Born approximation, the constraint on which the ensemble Monte Carlo method strongly relies.

In this respect the EMC method, through the calculations of the spin relaxation times, can give a test bed for modeling the many-body interactions in a semiconductor. Our simulations have demonstrated a very high sensitivity of the spin dephasing to the accuracy of the modeling of the electron-electron interactions.

\section{ACKNOWLEDGMENTS}

We acknowledge support from EPSRC Grant No. $\mathrm{EP} / \mathrm{F} 016719 / 1$. We wish to thank the reviewer for pointing out some important references of the vast EMC literature.

\footnotetext{
* gionnimarchetti@ gmail.com

$\dagger$ matthew.hodgson@york.ac.uk

irene.damico@york.ac.uk

1 S. D. Bader and S. S. P. Parkin Annual Review of Condensed Matter Physics, vol. 1, pp. 71 - 88, 2010.

${ }^{2}$ Y. G. Kusrayev Spin phenomena in semiconductors: physics and applications, vol. 53, pp. 725 - 738, 2010.
}

3 J. M. Kikkawa and D. D. Awschalom Phys. Rev. Lett., vol. 80, pp. 4313-4316, 1998.

${ }^{4}$ M. M. Glazov and E. L. Ivchenko JETP Letters, vol. 75, pp. 403-405, 2002.

5 M. M. Glazov and E. L. Ivchenko Journal of Superconductivity, vol. 16, p. 735, 2002.

6 J. H. Jiang and M. W. Wu Phys. Rev. B, vol. 79, p. 125206, 
Mar 2009.

7 S. Oertel, J. Hübner, and M. Oestreich Applied Physics Letters, vol. 93, no. 13, p. 132112, 2008.

${ }^{8}$ M. Römer, H. Bernien, G. Müller, D. Schuh, J. Hübner, and M. Oestreich Phys. Rev. B, vol. 81, p. 075216, 2010.

9 M. I. Dyakonov, Spin Physics in Semiconductors. Berlin Heidelberg: Springer- Verlag, 2008.

${ }^{10}$ M. S. Dresselhaus, G. Dresselhaus, and A. Jorio, Group Theory: Application to the Physics of Condensed Matter. Berlin Heidelberg: Springer-Verlag, 2008.

11 S. Kapoor, G. Marchetti, F. Rossi, and I. D'Amico, "Proceedings of the Joint European Magnetic Symposia - JEMS 2010," Journal of Physics: Conference Series, vol. 303, no. 1, p. $012095,2011$.

12 G. Marchetti, M. Hodgson, J. McHugh, R. Chantrell, and I. D’Amico Materials, vol. 7, no. 4, pp. 2795-2814, 2014.

13 C. Jacoboni and P. Lugli, The Monte Carlo Method for Semiconductor Device Simulation. Wien New York: SpringerVerlag, 1989.

14 M. V. Fischetti and S. E. Laux Phys. Rev. B, vol. 38, pp. 9721-9745, Nov 1988.

15 S. Saikin, Y. V. Pershin, and V. Privman IEE ProceedingsCircuits, Devices and Systems, vol. 152, no. 4, pp. 366-376, 2005.

16 M. Combescot Solid State Communications, vol. 65, no. 10, pp. $1221-1225,1988$.

17 C. A. Kukkonen and H. Smith Phys. Rev. B, vol. 8, pp. 46014606, Nov 1973.

18 C. J. Joachain, Quantum Collision Theory. Amsterdam: North-Holland Physics Publishing, 1987.

19 G. Giuliani and G. Vignale, Quantum Theory of Electron Liquid. Cambridge: Cambridge University Press, 2005.

${ }^{20}$ B. K. Ridley J. Phys. C: Solid State Phys., vol. 10, p. 1589, 1977.

21 B. K. Ridley, Quantum Processes in Semiconductors. Oxford: Oxford University Press, 2000.

22 J. Fabian, A. Matos-Abiague, C. Ertler, P. Stano, and I. Zutic Acta Phys. Slov., vol. 57, pp. 565-907, 2007.

23 J. Fu, M. Weng, and M. Wu Physica E: Low-dimensional Systems and Nanostructures, vol. 40, no. 9, pp. 2890 - 2893, 2008.

24 P. Y. Yu and M. Cardona, Fundamentals of Semiconductors. Berlin: Springer, 2010.

25 G. D. Mahan, Many-Particle Physics. New York: Kluwer Academic, 2000.

26 B. A. Sanborn Phys. Rev. B, vol. 51, pp. 4601-4606, Nov 1995.

27 D. Chattopadhyay and H. J. Queisser Rev. Mod. Phys., vol. 53, pp. 745-768, Oct 1981.

28 R. B. Dingle Philos. Mag., vol. 46, pp. 4601-4606, Nov 1955.

${ }^{29}$ N. W. Ashcroft and M. D. Mermin, Solid State Physics. Philadelphia: Saunders College, 1976.

30 D. Pines and D. Bohm Phys. Rev., vol. 85, pp. 338-353, Jan 1952.

${ }^{31}$ P. Lugli and D. Ferry Physica $B+C$, vol. 117, Part 1, no. 0, pp. $251-253,1983$.

32 P. Lugli and D. Ferry Physica $B+C$, vol. 129, no. 13, pp. 532 $-536,1985$.

33 M. E. Peskin and D. V. Schroeder, An Introduction to Quantum Field Theory. Westview Press, 1995.

${ }^{34}$ L. I. Schiff, Quantum Mechanics. Singapore: McGraw-Hill, 1968.

35 J. M. Ziman, Principles of the Theory of Solids. Cambridge: Cambridge University Press, 1972.

36 A. Mošková and M. Moško Phys. Rev. B, vol. 61, pp. 30483059, Jan 2000.

37 We can think the total cross-section as an effective area (geometrical cross-section) of a sphere seen by the colliding carrier at a given energy. Notice that the total cross-section may vary greatly even in a small energy range as indeed happens for a Yukawa potential.

38 T. G. V. der Roer and P. Widdershoven J. Appl. Phys., vol. 59, pp. 338-353, 1985.

39 Note that Eq. 97 does no longer depend on the impact parameter b: in the EMC scattering rates there is no angular dependence and consequently no dependence upon the impact parameter.

40 The third-body rejection method is already implemented in several EMC simulation programs, e.g. IBM's DAMOCLES and UIUC's Moka.

41 M. Pugnet, J. Collet, and A. Cornet Solid State Commun., vol. 38, pp. 338-353, 1981.

42 Mayer-Bartoli's phase-shift analysis has also been implemented in IBM's DAMOCLES program.

43 J. R. Meyer and F. J. Bartoli Phys. Rev. B, vol. 23, pp. 54135427, May 1981.

44 If $E_{\text {lab }}$ denotes the energy of an electron $A$ in the laboratory frame of reference colliding with an electron $B$, then the energy $E_{r e l}$, available for collision in the center of mass frame of reference, is given by $E_{r e l}=m_{B} /\left[\left(m_{A}+m_{B}\right) E_{l a b}\right]$, i.e., $E_{r e l}=0.5 E_{l a b}$ for the case of the e-e scattering ${ }^{34}$. Notice that the two colliding carrier masses may be slighlty different due to the nonparabolicity of the conduction band. However this has in general a negligible effect on the previous consideration.

45 J. R. Meyer and F. J. Bartoli Phys. Rev. B, vol. 28, pp. 915926, Jul 1983.

${ }^{46}$ Notice that the e-e scattering formula we use ${ }^{12}$, has a slightly more complicated dependence upon the electron density, as $n_{e}$ enters also into the variable $\beta_{\mathrm{TF}}$. However in first approximation we can assume that $w_{\text {ee }} \propto n_{\mathrm{e}}$.

47 N. P. Heydenburg and G. M. Temmer Phys. Rev., vol. 104, pp. 123-134, Oct 1956.

48 A. Mošková and M. Moško Phys. Rev. B, vol. 49, pp. 74437452, Mar 1994.

49 With regards to this formula $\sqrt{18}$, we recall that it describes the unpolarized scattering probability of fermions, averaging over the possible triplet and singlet spin states which they can form. Then some care should be taken to implement this condition. 